\title{
El Diplomado en Formación de Profesores de Español como Lengua Extranjera (DFPELE).
}

\section{La primera experiencia del CEPE en el diseño de un curso en línea}

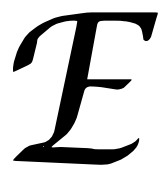

$n$ febrero de 2003, el Centro de Enseñanza para Extranjeros (CEPE), en colaboración con la Secretaría de Relaciones Exteriores (SRE), comenzó a impartir el Diplomado en Formación de Profesores de Español como Lengua Extranjera. Se trata de un diplomado que se cursa en línea. El objetivo de este artículo es ofrecer un panorama contextualizador de este proyecto, explicar su fundamentación pedagógica, y compartir la experiencia de los docentes del CEPE como diseñadores de contenido y como tutores a distancia.

\section{Martha Jurado Salinas}

\section{Introducción}

El manejo de lenguas adicionales y de nuevas tecnologías se han convertido, en la actual sociedad de la información, en herramientas indispensables en la formación de recursos humanos.

Dentro de este contexto, el español representa una opción atractiva ya que se encuentra en un proceso de clara expansión debido a razones no sólo de índole demográfica, sino también al empuje provocado por el gran aumento de personas que, por muy diversos motivos, lo han elegido como segunda lengua.

Por otro lado, las nuevas tecnologías de la información y comunicación (computadoras, equipos multimedia, redes locales, Internet, TV digital etc.) que podríamos definir como sistemas y recursos para la elaboración, almacenamiento y difusión digitalizada de información basados en el empleo de tecnología informática, están provocando profundos cambios y transformaciones de naturaleza económica, social y cultural. Muchos pensadores e investigadores denominan a este nuevo con- texto social, cultural, político y económico en el que vivimos como "sociedad de la información". La información se ha convertido en una materia prima que se elabora, se transforma y se comercializa como cualquier otro producto manufacturado. La "información" ha impregnado tanto las instituciones y organizaciones sociales como la vida cotidiana de cada individuo de tal modo que, inevitablemente, todas las personas producimos y consumimos diariamente información ya sea a través del teléfono, de los medios de comunicación o de las computadoras.

El Diplomado en Formación de Profesores de Español como Lengua Extranjera (DFPELE) es un proyecto educativo que tiene como propósito formar recursos humanos que satisfagan la creciente demanda de profesionales bien preparados en los aspectos lingüísticos, didácticos y actitudinales relacionados con la enseñanza del español como lengua extranjera. El DFPELE es parte del cambio que aprovecha además las nuevas tecnologías, ya que se trata de un diplomado que se cursa básicamente a cavés de Internet (http://diplomados.cepe.unam.mx/diplomados.html). 
Así pues, el DFPELE conjuga las dos herramientas básicas de la formación de recursos humanos del nuevo siglo, en un proyecto que pretende acercar al estudiante al mundo laboral a través de contenidos interrelacionados cuya calidad y variedad se basan en la primacía del conocimiento sobre la información, la creatividad, la capacidad de trabajo en grupo, de acuerdo, de cooperación y de tolerancia; siempre en convivencia con las habilidades clásicas como la lectura y escritura.

\section{I.1. Antecedentes institucionales}

La creciente importancia que la lengua española tiene en la actualidad por motivos de índole demográfica, económica, política y cultural, ha propiciado que, en diversos países hispanohablantes, instancias gubernamentales del más alto nivel muestren interés en proyectos relacionados con su preservación y difusión.

En nuestro país, la Secretaría de Relaciones Exteriores, a través del Instituto de México, asumió en el año 2000 la tarea de difundir la enseñanza del español en el extranjero. Para lograr tal objetivo, se acercó al CEPE, una institución con más de 70 años de experiencia en esa labor. La SRE apoyó económicamente el proyecto de diseño y puesta en operación del Diplomado en Formación de Profesores de Español como Lengua Extranjera, cuyo antecedente inmediato fue el Curso de formación de profesores de español a no hispanohablantes que se ha impartido en el CEPE desde 1995 y que ha contribuido a formar profesionales en la enseñanza del español que actualmente laboran en diversas instituciones educativas privadas y públicas, nacionales y extranjeras. La modalidad a distancia diseñada con el apoyo de la SRE tiene como finalidad atender las necesidades de personas, tanto mexicanas como extranjeras, interesadas en formarse como profesores de español, que no tienen oportunidad de tomar cursos presenciales.

Así pues, la SRE y la UNAM se unieron en este esfuerzo que se espera tenga un efecto multiplicador, ya que, al contar en distintos puntos geográficos con profesores bien preparados, se podrá atender en un futuro de manera más adecuada la demanda de clases de español, contribuyendo así a la difusión y preservación de nuestra lengua.

\section{Proceso de diseño del diplomado en línea}

Con el apoyo de la SRE, ocho profesores del CEPE durante seis meses, se dedicaron de tiempo completo al proyecto. Éste se inició en el mes de enero de 2002, con la capacitación de los profesores en un curso de 20 horas sobre Comunidades de Aprendizaje ofrecido por la CUAED (Coordinación de Universidad Abierta y Educación a Distancia); y prosiguió, de abril a julio, con dos talleres impartidos por personal de la DGSCA (Dirección General de Servicios de Cómputo Académico): Planeación y desarrollo de contenidos de páginas educativas en Internet (30 horas) y Aspectos básicos de creación de páginas $W E B$ educativas (40 horas).

La formación de los profesores como diseñadores de contenidos implicó paralelamente su integración con un equipo multidisciplinario conformado además por cuatro técnicos informáticos, cuatro programadores de sistemas, un diseñador gráfico, dos diseñadoras instruccionales, así como la asesoría permanente del equipo del Centro de Apoyo a la Docencia del CEPE. 
El diseño formativo o educativo ${ }^{1}$ del DFPELE fue un proceso de trabajo interdisciplinario que comprendió las siguientes etapas:

a) Análisis de las necesidades de aprendizaje y del contexto donde el proyecto se ininserta;

b) Diseño:

1. Definición de los objetivos de formación;

2. Selección de los recursos didácticos y tecnológicos más adecuados teniendo en cuenta los procesos de aprendizaje;

3. Desarrollo de los contenidos y las actividades;

4. Pilotaje;

5. Diseño y aplicación de instrumentos de evaluación para dar seguimiento al proyecto;

6. Reestructuración y actualización como procesos constantes.

El análisis de necesidades se realizó con base en una búsqueda y análisis de información respecto a la expansión de la lengua española en el mundo actual y a la oferta educativa de cursos en línea de formación de profesores de español como L/E (lengua extranjera) ${ }^{2}$. Con base en los objetivos de formación, se elaboró un perfil de egreso ${ }^{3}$, el cual quedó definido en los siguientes términos:
El egresado del DFPELE es un individuo que posee amplios conocimientos sobre el sistema de la lengua española y sabe transmitirlos. Considera la lengua como un elemento ordenador del pensamiento, como un instrumento de comunicación que permite no sólo el empleo de formas y estructuras lingüísticas, sino también su uso en diferentes contextos comunicativos.

Es un individuo apto para ofrecer una respuesta innovadora a las demandas educativas en el campo de la enseñanza de la lengua española mediante la aplicación de los conocimientos, habilidades y destrezas adquiridas durante el diplomado.

Es un individuo con una amplia visión del mundo, con actitudes positivas y de comprensión y tolerancia hacia otros individuos, capaz de responsabilizarse de su desarrollo profesional, con disposición para enseñar cada vez mejor y con capacidad para reflexionar sobre su propia labor docente como elemento esencial para desarrollar el conocimiento y las teorías didácticas.

Para la selección de recursos, se trabajó con base en la elaboración de un guión técnico; es decir, una estructura general que permitió crear el diseño específico. En el guión se de-

\footnotetext{
'The Applied Research Laboratory de la Penn State University define el concepto de diseño formativo y de tecnología de la formación asi: "Definimos el instructional design como el desarrollo sistemático de una acción formativa basado en las teorias de aprendizaje, con el fin de asegurar la calidad de la formación. Es el proceso global de análisis de necesidades educativas, de determinación de los objetivos de aprendizaje derivados de estas necesidades y de la definición del soporte y los medios que hay que utilizar en el desarrollo de la acción a fin de que se alcancen los objetivos. Incluye el desarrollo de materiales didácticos y actividades de aprendizaje, así como el proceso de evaluación tanto del material en sí como de los procesos de aprendizaje de los usuarios." "(Lourdes Guardia. "El diseño formativo: un nuevo enfoque de diseño pedagógico de los materiales didácticos en soporte digital", en _Aprender en la virtualidad, Duart y Sangrá p. 173).

${ }^{2}$ Para la discusión del primer punto se tomó como base la ponencia presentada en el II Congreso Internacional de la Lengua Española: Jurado, M. y G. Pulido, "La enseñanza del español en México. Una industria en Potencia", Valladolid, 2001. Se analizó también la oferta educativa en el terreno de la formación de profesores, principalmente, los cursos ofrecidos por las Universidades de Barcelona, Alcalá y Antonio de Nebrija.

${ }^{3}$ Es un individuo con una amplia visión del mundo, con actitudes positivas y de comprensión y tolerancia hacia otros individuos, capaz de responsabilizarse de su desarrollo profesional, con disposición para enseñar cada vez mejor y con capacidad para reflexionar sobre su propia labor docente como elemento esencial para desarrollar el conocimiento y las teorías didácticas.
} 
finieron los aspectos del diseño (concepto del sitio, uso de escudos, logotipos, colores, etc.); los elementos de las páginas principal y de materia (encabezado, barras de navegación y área de contenido); las características formales del área de contenido (fondo, títulos, subtítulos, etc.); las herramientas y sus funciones (rincón del profesor, rincón del alumno, mis notas, carpeta de trabajo, foro, chat, avisos, calendario, registro de evaluaciones, buscador y ayuda).

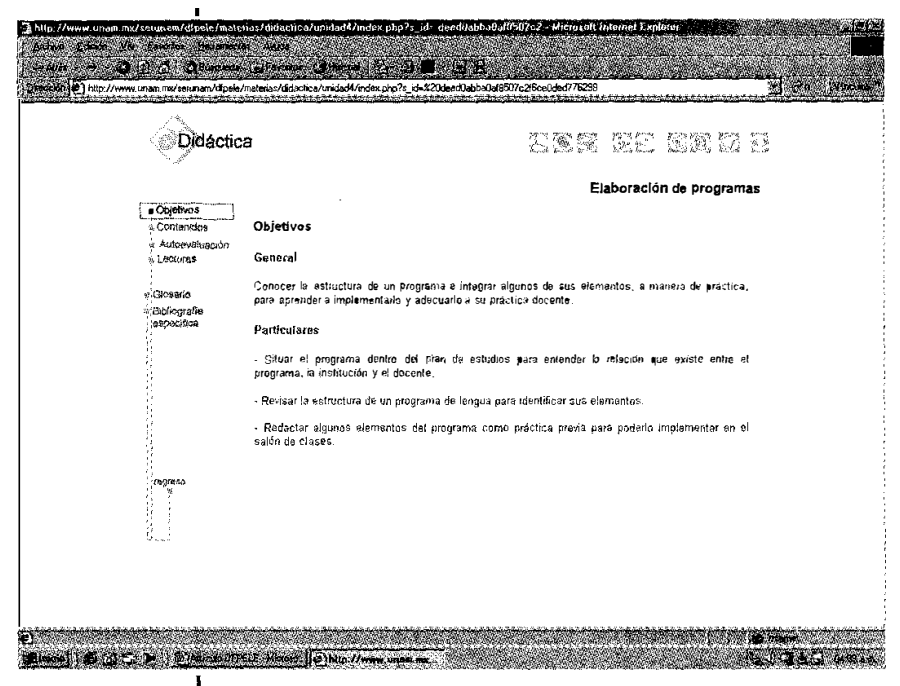

Paralelamente, cada experto en contenido diseñó para su materia la presentación, objetivos, metodología, criterios de evaluación, calendarización y bibliografía general; para cada unidad, a su vez, fueron diseñados objetivos (generales y particulares), contenidos, actividades, autoevaluación y bibliografía específica. Esta labor implicó la definición de la

plataforma multimedia apropiada a los contenidos (texto, audio, ilustraciones, animaciones, video, etc.).

Se trató de atender la diversidad de motivaciones, necesidades y estilos de aprendizaje suministrando en los materiales didácticos contenidos básicos, con orientación biblio-

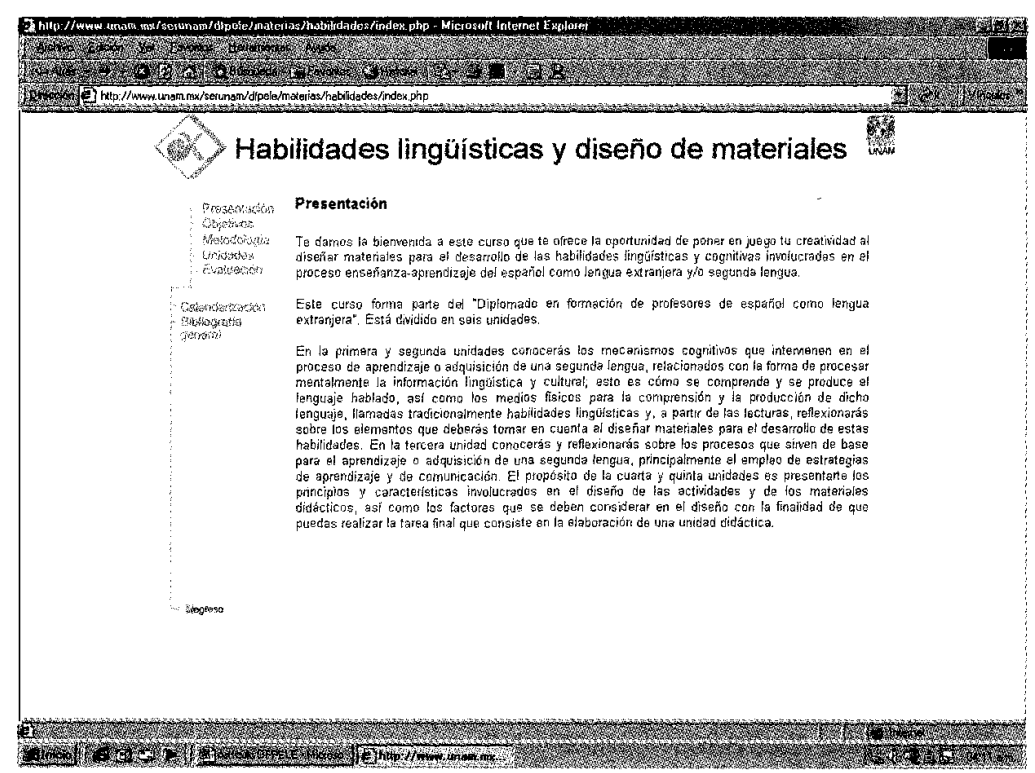


gráfica que posibilitara el reforzamiento y profundización en algunos de ellos (el pago de derechos de autor limitó la inclusión de lecturas originales). Asimismo, se trató de tener presentes las diferentes maneras de estudiar, empleando distintos grados de complejidad en las estrategias de trabajo, utilizando lenguajes variados, glosarios con enlaces hipertextuales y estilo próximo al estudiante.

Para la elaboración de los materiales didácticos, los expertos en contenido siguieron las siguientes pautas:

* Presentar primero los contenidos generales y más simples y después los más complejos y diferenciados.

* Empezar cada materia y cada unidad con una presentación que proporcionara una visión global y general del tema, y después pasar a un análisis de las partes para, finalmente, proponer una recapitulación a través de una actividad de autoevaluación.

* Mostrar las relaciones entre los contenidos, incluyendo los de asignaturas diferentes.

* Partir de núcleos temáticos o de temas próximos a la práctica docente.

* Recordar y repasar contenidos anteriores relacionados con el tema.

* Dar pautas para analizar y establecer relaciones entre contenidos.

Un aspecto fundamental en el diseño de los materiales fue la cuantificación y desarrollo de las actividades de aprendizaje necesarias para alcanzar los objetivos propuestos. Atendiendo a un concepto de aprendizaje como proceso de construcción creativa que busca propiciar en los alumnos la adquisición de habilidades para el autoaprendizaje y para el aprendizaje colaborativo, se trató de diseñar actividades que desarrollaran diferentes es- trategias cognitivas (comparar, clasificar, inducir, deducir, abstraer, analizar, etc.); que activaran conocimientos previos; que orientaran el aprendizaje; que estimularan la participación; que proporcionaran retroalimentación automatizada o del profesor o bien del grupo; que permitieran un seguimiento del proceso y la evaluación del rendimiento; y finalmente, que motivaran o provocaran curiosidad por lo que se aprende. La tarea fue ardua, pero, en conjunto, las actividades diseñadas cumplieron con estos requisitos en mayor o menor medida.

Cabe destacar que algunos de los expertos, como producto de la capacitación recibida en los talleres, trabajaron los contenidos también en formato $\mathrm{btml}$, lo cual significó un aprendizaje adicional, que redundó en una mejor comprensión del medio, con el consecuente mejoramiento en la calidad de los materiales. Otro punto rescatable fue la discusión para alcanzar consensos. El llegar a acuerdos sobre cada uno de los puntos del diseño educativo implicó un gran trabajo de tolerancia, capacidad para escuchar y respeto hacia la opinión de los otros, lo cual enriqueció significativamente nuestras relaciones laborales.

\section{Descripción del diplomado y del resultado esperado}

El resultado de un año de trabajo interdisciplinario fue la versión del Diplomado en Formación de Profesores de Español como Lengua Extranjera que se empezó a pilotear en febrero de 2003.

El DFPELE está dirigido a licenciados o titulados en estudios superiores con o sin experiencia previa en la enseñanza de $E / L E$ que deseen adquirir las habilidades necesarias para desarrollar su formación como docen- 
tes de $E / L E$. Se trata de un programa que se cursa fundamentalmente en línea e incluye una práctica y presentación de un trabajo final, las cuales se llevan a cabo al finalizar las materias teóricas, en la sede del CEPE en la ciudad de México.

Tiene como objetivo formar profesionales de la enseñanza del español como lengua extranjera, conocedores del sistema lingüístico en todos sus niveles y capaces de desarrollar estrategias didácticas innovadoras.

El diseño curricular del diplomado conjuga conocimientos teóricos y prácticos alrededor de dos ejes fundamentales: sistema y uso de la lengua y habilidades didácticas; más un tercero que subyace a todo el currículo (actitudes y valores). Está estructurado en diez materias (450 horas) que podrán cursarse en un año:

\begin{tabular}{|c|c|c|}
\hline & Materias & $\begin{array}{l}\text { Mo. de } \\
\text { horas }\end{array}$ \\
\hline & $\begin{array}{l}\text { 1. Fonología y fonética } \\
\text { aplicada }\end{array}$ & 30 \\
\hline 2 & 2. Linguística general & 45 \\
\hline & 3. Morfosintaxis & 45 \\
\hline & 4. Didáctica & 45 \\
\hline & $\begin{array}{l}\text { 5. Aspectos gramaticales } \\
\text { problemáticos de } \\
\text { la enseñanza del español }\end{array}$ & 60 \\
\hline & $\begin{array}{l}\text { 6. Habilldades lingüísticas y } \\
\text { diseño de materiales }\end{array}$ & 60 \\
\hline & $\begin{array}{l}\text { 7. Variantes dialectales del } \\
\text { español estándar }\end{array}$ & 45 \\
\hline & $\begin{array}{l}\text { 8. La tecnología en la } \\
\text { enseñanza de lenguas }\end{array}$ & 30 \\
\hline & 9. Evaluación & 45 \\
\hline & $\begin{array}{l}\text { 10. Planeación, observación } \\
\text { y práctica de clase }\end{array}$ & 45 \\
\hline
\end{tabular}

La formación resultante posee dos vertientes: conocimientos y habilidades.
Conocimientos del sistema lingüístico (para ser enseñado a no hispanohablantes), así como de teorías metodológicas, didácticas y de evaluación de enseñanza de lenguas, lo cual se traduce en dominio de:

* Conocimientos descriptivos y normativos sobre el sistema de la lengua en distintos niveles: fonético, morfosintáctico, semántico y pragmático.

* Conocimientos sobre las distintas variantes dialectales del español.

* Metodología de la enseñanza de lenguas y su evolución.

* Conceptos y criterios fundamentales de la evaluación del proceso de aprendizaje.

La segunda vertiente tiende a la formación de docentes con disposición didáctica y metodológica que propicien en el alumno el desarrollo eficaz de las competencias lingüística y comunicativa, el cual logra mediante su habilidad para:

* Proponer objetivos y contenidos lingüísticos, comunicativos y estratégicos suministrando información necesaria y adecuada al nivel de lengua de los estudiantes.

* Identificar y aplicar de manera pertinente y apropiada distintos métodos de enseñanza.

* Diseñar y organizar actividades en el aula que promuevan la aplicación de estrategias de aprendizaje y comunicación en los alumnos extranjeros en relación con las cuatro habilidades lingüísticas (hablar, escribir, leer y escuchar), y que se integren en el diseño de tareas. Esta destreza didáctica implica desarrollar en el alumno la autonomía, el descubrimiento de sus procesos y estilos particulares de aprender, 
la actitud positiva ante el error como parte del proceso, la confianza en sí mismos al practicar la lengua meta, la reflexión sobre la lengua como sistema formal y el comportamiento interactivo, colaborador y negociador.

* Diseñar materiales aplicando los conocimientos adquiridos durante el diplomado .

* Seleccionar materiales y recursos didácticos para utilizarlos o adaptarlos de manera creativa y adecuada.

* Elaborar y aplicar una metodología de evaluación que le permita, con base en un sistemático ejercicio de reflexión y análisis, recibir retroalimentación para reestructurar los distintos aspectos de su práctica docente.

Finalmente, el tercer eje que recorre el currículo, el de actitudes y valores, tiende a la formación de docentes que valoren su actividad como una profesión, lo cual implica, entre otros muchos aspectos, responsabilidad para alcanzar la más actualizada preparación posible, disposición para enseñar cada vez mejor y capacidad para reflexionar sobre su propia labor docente.

\section{Algunas reflexiones sobre la experiencia de los profesores como elaboradores de contenidos y como tutores a distancia}

Aunque éste es un tema tratado con mayor amplitud en otros documentos elaborados por los profesores participantes en el proyecto $^{4}$, no quiero dejar de mencionar que existe entre los autores-tutores el consenso de que la experiencia de haber elaborado los contenidos redundó en una mejora sustancial de nuestra práctica docente en general. Es decir, además del aprendizaje instrumental que significó la elaboración de las páginas WEB, el haber tenido que pensar en términos como "objetivos generales y específicos", "exposición lecturable, "palabras clave", "actividades que propiciaran la aplicación o análisis de la información" etc. nos llevó a replantear nuestro trabajo en el aula y a utilizar con mayor conocimiento de causa algunos de los recursos que las nuevas tecnologías ofrecen.

Por otra parte, la experiencia como tutores en línea que hasta ahora no todos los diseñadores de contenido han tenido la oportunidad de vivir nos obligó a cambiar el papel protagónico de transmisor de conocimientos que muchos nos resistíamos todavía a abandonar del todo para asumir plenamente el de "facilitador", es decir, de orientador que guía para que el estudiante desarrolle su propio proceso de aprendizaje, a la vez que le ofrece retroalimentación, dinamiza su proceso de aprendizaje y atiende sus dudas y necesidades.

Resultaron particularmente arduas, quizá por lo inéditas que eran para nosotros, las labores de calendarización de actividades (había que imaginar cuánto tiempo le tomaría a un estudiante realizar la actividad, su habilidad en el manejo de la computadora, la capacidad de su equipo, etc.), el seguimiento de las actividades y de las participaciones en los foros, así como

${ }^{4}$ Cf. Rosa Esther Delgadillo, "Características de la figura del tutor en el curso en línea", ponencia presentada en las mesas de diag nóstico local Rumbo al Congreso Universitario de la UNAM, mayo de 2003; Eva Campos, participación "Un curso en línea, descripción de una experiencia", dentro del panel "Modelos de formación docente a distancia: la experiencia CELE, CEPE, ENEPAcatlán", 11 Encuentro Nacional de Profesores de Llenguas Extranjeras. CELE-UNAM, agosto de 2003; Ma. del Carmen Koleff, "Formación de profesores de español vía Internet", $11^{\circ}$. Encuen Nacional de Profesores de Lenguas Extranjeras CELE-UNAM, agosto de 2003. 
la moderación de los chats; todo ello aunado a algunas pequeñas dificultades técnicas que, como es natural, se presentaron en el pilotaje. Después de nuestra primera experiencia, el consenso fue que el trabajo en línea resultó más demandante que nuestra labor en el aula de manera presencial.

\section{Pilotaje y evaluación de la primera fase}

En febrero de 2002, comenzaron a ofrecerse las dos primeras materias del DFPELE: Lingüística teórica y aplicada, y Fonología y fonética aplicada. Dieciocho aspirantes llenaron solicitud; diez pagaron y presentaron el examen de admisión: nueve de ellos residen en Estados Unidos (incluyendo a una ecuatoriana, una argentina y a un venezolano) y uno en Canadá. Todos ellos fueron admitidos. De los diez estudiantes que se inscribieron, dos abandonaron el curso durante las dos primeras materias. Al terminar el segundo bloque, se registró un abandono más. A mediados del tercer bloque, una alumna que había cursado el primer módulo de manera presencial, se incorporó a la modalidad a distancia. En total quedaron ocho estudiantes.

Aunque hay diferencias importantes (edad, lugar de residencia, formación previa y situación personal) todos coinciden en ser profesionistas y hablantes de español. Resalta el hecho de que algunos ya tienen experiencia enseñando español, pero necesitan actualizar sus conocimientos ya sea para fortalecer su actividad docente o bien para cumplir con un requisito que les impone la institución donde trabajan.

Como primera fase de validación del proyecto, en julio de 2003, se aplicó una encuesta dirigida a los estudiantes. Se valoró su sentimiento de satisfacción respecto a los conte- nidos, materiales, actividades, dinámica de trabajo, comunicación, actuación de los tutores, duración de los bloques, integración del grupo, autoevaluación, proceso de inscripción, funcionamiento del sitio, diseño de interfaz, diseño gráfico y desempeño de los contactos académico-administrativo y técnico.

Según la evaluación aplicada a las cuatro materias que hasta julio se habían ofrecido en su totalidad, el resultado es alentador: existe unanimidad respecto a la calidad y eficacia de los aspectos formales y técnicos del sitio; respecto a los contenidos y la actuación de los tutores el rango se mantiene entre "excelente" (o siempre) y "bueno" (o "casi siempre"). Aunque también se manifestó una opinión unánime de que el Diplomado cumple con sus expectativas, una de las alumnas mencionó que eran necesarias algunas modificaciones, pero no especificó cuáles.

Con base en los resultados arrojados por la evaluación, el equipo interdisciplinario llegó a la conclusión de que en general el DFPELE cumple con los lineamientos que nos habíamos propuesto; sin embargo, es necesario seguir trabajando en aspectos como el desarrollo de habilidades, las actitudes, la motivación, la interacción entre los actores y con los materiales, y la creación de entornos virtuales colaborativos. Por ello, se determinó pertinente realizar los siguientes ajustes:

* Revisar la relación entre el tiempo de dedicación previsible y el real para proponer un ritmo de trabajo que garantice la mejor consecución de los objetivos pretendidos, tanto por parte de los alumnos como de los tutores.

* Equilibrar mejor la relación entre flexibilidad y rimo de trabajo. Revisar el itinerario para ajustarlo a las necesida- 
des de los alumnos, sin que tengan que seguir estrictamente la secuencia recomendada, si no es necesario. Se trata de abrir y flexibilizar más la metodología sin que ello afecte la calidad académica.

* Hacer mayor énfasis en el desarrollo de habilidades y de herramientas para la formación continua.

* Diversificar las estrategias de trabajo colaborativo para realmente conseguir la creación de comunidades virtuales de aprendizaje, ello implica también intensificar el apoyo personalizado para conseguir mayor grado de implicación y de motivación.

* Continuar la capacitación tanto pedagógica como técnica de los tutores, para conocer con mayor profundidad los medios, sus posibilidades y convenciones y consecuentemente, mejorar los materiales y el desempeño docente .

* Elaborar guías de validación que orienten la producción de materiales con esquemas y estructuras similares. Contar con guías de aplicación didáctica ${ }^{5}$, de aplicación tecnológica y con un libro de estilo en WEB, nos proporcionaría las bases para garantizar la continuidad del proyecto.

* Realizar, al concluir el primer pilotaje, una evaluación externa que certifique la calidad académica del DFPELE.
Así pues, la retroalimentación aportada por la evaluación nos permitirá hacer los ajustes necesarios para la segunda generación que se abrió en septiembre de 2003.

\section{Conclusiones}

La participación en el diseño educativo y en la aplicación del DFPELE nos brindó la oportunidad de vivir una experiencia de interdisciplinariedad, interculturalidad (porque había una profesora española) y desarrollo profesional precisamente en los ejes que habíamos planteado como base del proyecto: conocimientos, habilidades y actitudes. Profundizamos conocimientos sobre las materias que impartimos y aprendimos a diseñarlas pedagógicamente aprovechando las posibilidades de comunicación e interacción que brinda la tecnología; desarrollamos habilidades en el manejo de la computadora y, finalmente, tuvimos que aprender a poner en práctica actitudes de tolerancia y respeto en el trabajo en equipo.

Este primer esfuerzo que tan arduo resultó tiene como gratificación el habernos enseñado cómo trabajar futuros proyectos: se trata de integrar los ámbitos disciplinario, metodológico y tecnológico contando con el apoyo conjunto de instituciones. Porque ahora sabemos que el DFPELE constituye sólo el primer paso. El siguiente será pensar en su integración en un currículo progresivo

\footnotetext{
${ }^{5}$ Coincidimos en este sentido con la opinión de Gabriel Ferrate: "... si queremos que nuestra sociedad sea una sociedad del conocimiento y no solamente de la información, será necesario trabajar desde un enfoque pedagógico en el cual la creación de comunidades de aprendizaje virtuales, el tratamiento de la información, la generación de nuevas estrategias de comunicación y de aprendizaje sean imprescindibles y llevados a cabo por profesionales preparados.

Estos profesionales se especializan en la creación de ambientes de trabajo cada vez más simples e intuitivos, estudian las posibilidades de la tecnología multimedia para ofrecer un aprendizaje más motivador y eficaz, optimizan las posibilidades comunicativas y el trabajo cooperativo, introducen la interactividad en el proceso y se convierten, pues, en unos especialistas del diseño formativo y de la docencia en sistemas de formación virtuales" ["Prólogo" a Aprender en la virtualidad. Duart y Sangrá (comp), p. 10]. "Esta guía proporcionaría información sobre el entorno donde ha de actuar el material (características de los estudiantes, de la institución, modelo pedagógico, entorno virtual de aprendizaje, etc.). Establecería también la metodología y los criterios para la elaboración de los materiales didácticos (ratamiento de la bibliografía y recursos, reproducción de textos u oros materiales, criterios tipográficos, etc.).
} 
que contenga como primera instancia cursos "a la carta" que satisfagan necesidades inmediatas y prácticas de los docentes, por ejemplo, de técnicas de comprensión de lectura, de enseñanza de la lengua escrita, de manejo de la voz, etc. El DFPELE sería un "menú completo" intermedio para aquellos que estén dispuestos a continuar su formación profundizando en ciertos contenidos. Finalmente, el objetivo a futuro sería el diseño en línea de una Maestría en enseñanza de español y cultura mexicana.

Se trata de un reto que hemos asumido porque como universitarios, estamos comprometidos con una educación que forme profesionales con capacidades que les permitan desempeñarse en un mercado laboral sujeto a las exigencias de la actual era de la información.

\section{Bibliografía}

Arrarte, Gerardo y José Ignacio Sánchez de Villapadierna, Internet y la enseñanza del español, Madrid, Arco/Libros, 2001 (Cuadernos de didáctica del español/LE).

Cruz Piñol, Mar, Enseñar español en la era de Internet, Barcelona, Octaedro, 2002 (Recursos 41).

Duart, Joseph M. y Albert Sangrá, "Aprendizaje y virtualidad: ¿un nuevo paradigma formativo?", en Aprender en la virtualidad, Barcelona, Gedisa, 2000. (Edicions de la Universitat Oberta de Catalunya).

Guardia, Lourdes. "El diseño formativo: un nuevo enfoque de diseño pedagógico de los materiales didácticos en soporte digital", en Aprender en la virtualidad, Duart y Sangrá, Barcelona, Gedisa, 2000, (Edicions de la Universitat Oberta de Catalunya), pp. 171-187.

Sangrá, Albert. "Materiales en la web. Un proceso de conceptualización global", en Aprender en la virtualidad, Barcelona, Gedisa,
2000. (Edicions de la Universitat Oberta de Catalunya).

Agradecimiento especial a José Luis López, Rosa Esther Delgadillo, Patricia Martínez, Yolanda Uribe, Eva Campos, Sandra Cruz, Rebeca Valenzuela y Mercedes Jódar, por sus valiosas observaciones. 